radiation of the prostate that organisms permeate from its interior into the space from which the gland has been removed. The raw prostatic bed is a suitable incubator, and violent sepsis may result. I have had experience of two such cases. Thus we learn the necessity of caution in dealing with a primary focus. After the onset of general infection, abscesses may form in any situation ; their discovery and evacuation is often the signal of recovery.

Aseptic technique in the operating theatre may be of a high order, but the best efforts may be nullified if nurses and assistants in charge of, or in contact with, septic cases are part of the team. I have had a case of Streptococcus haemolyticus septicaemia after a simple appendicectomy which was traceable to a nurse who, when not in the theatre, was in charge of a septic gynaecological case in an adjoining room. Again, epidemics of septicaemia have been traced to the throats of seasonal carriers when there was laxity about masking all those within the reach of operative work. We cannot yet claim that prophylactic measures in surgery have reached perfection.

\section{REMARKS ON TREATMENT}

Surgeons, physicians, and biochemists see many cases of septicaemia without discoverable cause, and the treatment of this group is in consequence not satisfactory. What is the best line of treatment in a case with a positive blood culture? Assuming that there is no accessible primary focus, and that the organism is the streptococcus, serum should be employed. Fifty cubic centimetres of the univalent form given intravenously on three consecutive days has been suggested by Horder. The results of serum therapy justify immediate and adequate dosage. Should we employ intravenous injections of such substances as mercurochrome and gentian violet? The literature in favour of these and allied remedies is interesting. There are strong advocates for this line of treatment. My own practice has been to use mercurochrome, $20 \mathrm{c.cm}$. of a 1 per cent. solution intravenously on two or three consecutive days when everything else had been tried without success, but I am not convinced that intravenous injections of any antiseptic are useful. Horder recommends arsenic as the most useful drug. Colebrook states that in the treatment of syphilis by the salvarsan group the blood serum becomes a destructor of streptococci and that the leucocytes remain unaffected. There is therefore a prima facie case for the more extended use of arsenic in septicaemic conditions. Blood transfusions have little or no direct effect in the control of sepsis ; indirect benefit may be derived in the more chronic cases.

Large quantities of fluid given intravenously and by the rectal and subcutaneous routes are always beneficial, and 3,000 to $4,000 \mathrm{c} . \mathrm{cm}$. daily of 10 per cent. dextrose solution in normal saline may be given continuously by the drip method through a cannula in the internal saphenous vein without disturbance to the patient, or in divided doses through a needle into the veins of the arm. The patient is thus fed, the glycogen reserve of the liver is raised, the heart is stimulated, and diuresis is increased. Tap water can be administered simultaneously by the drip method into the rectum, or saline into the subcutaneous tissues of the breast. Such methods assist in the dilution and elimination of toxins. Murphy's arguments in favour of ample fluids were not confined to cases of septic peritonitis.

Finally, treatment of septicaemic cases in the open air when practicable should be employed. There is no comparison between the results of treatment of local sepsis in an open-air hospital and in a city hospital. In like manner, patients suffering from infection of the blood stream respond to treatment better in the open air.

\section{OCULAR COMPLICATIONS OF CHRONIC EPIDEMIC ENCEPHALITIS *}

BY

THEODORE H. WHITTINGTON, M.D., M.R.C.P., D.O.M.S.

CHIEF OPHTHALMIC ASSISTANT, KING'S COLLEGE HOSPITIL; OPHTHALMIC SURGEON, QUEEN MARY'S HOSPITAL AND SOUTH-EASTERN HOSPITAL FOR CHILDREN ; CONSULTING OCULIST, POST-ENCEPHALITIS UNIT, LONDON COUNTY COUNCIL

During the last six years I have been examining cases at the post-encephalitis lethargica unit of the London County Council, at the Northern Hospital, Winchmore Hill. It was felt, therefore, that some remarks on the ocular conditions met with, based on notes of 174 cases of the later stages of the disease in children, might be of interest, and that it might be well to review the subject briefly from the ophthalmological standpoint, in the light of more recent knowledge and longer experience.

Ophthalmologists are interested in epidemic encephalitis (or encephalitis lethargica, so-called) for many reasons, which can be summarized thus: (1) As regards the diagnosis of the disease, the ocular phenomena being of great importance in this respect, both in the acute and chronic stages. (2) On account of the peculiarity of these ocular signs, some of the most extraordinary of which occur only in this disease. (3) Because the correlation of these ocular phenomena with the known pathology of the disease helps to throw further light on the neurology of some of the ocular movements, and is closely related to the subject we have been discussing $\dagger$ in this Section-namely, ocular palsies. (4) Lastly, because the great benefit obtained in chronic encephalitic Parkinsonism by the use of the belladonna group of drugs is now well recognized. As a result, the ophthalmologist is called on to counteract the visual disabilities which may be produced by this treatment.

\section{Ocular Signs in the Acute Stage}

You scarcely need to be reminded of the importance of the ocular signs and symptoms of the acute stage, which result principally from varied kinds of ophthalmoplegia. There are the transient nerve-root paralyses, giving rise to transient diplopia, and, as I shall suggest, to the ptosis. There are the irregular ophthalmoplegias due to deeper lesions, resulting in altered movements of the eyeballs, defects in the movements of the pupils, and peculiarities in the movements of the lids. In the Ministry of Health Report, No. 11, 78 per cent. of the patients, in a series of 1,152 case reports, had the external ocular muscles affected.in some way or another. Thus, ptosis occurred in nearly 50 per cent., squint in 34 per cent., diplopia without obvious squint in 18 per cent., and nystagmus in 18 per cent.

In the acute stage epidemic encephalitis presents multitudinous symptoms, and may, and does, simulate many diseases of the brain. I would suggest, however, that there are two common types with which, particularly, the ophthalmologist may meet. "The first is a type showing ocular disturbances occurring in a febrile disease, with marked disorder of sleep. The second is that of a socalled "influenza" with diplopia-a milder type often missed, but equally liable to sequels.

It should also be added that the presence of papilloedema or of optic neuritis is decidedly against the diagnosis of encephalitis.

\section{Ocular Signs in the Chronic Stage}

One can contrast these ocular complications of the acute stage with those seen in the chronic stage. It has. been

* Paper read in the Section of Ophthalmology at the Annual Meeting of the British Medical Association, Eastbourne, 1931.

$\dagger$ See report, British Medical Journab, August 8th, 1931 (p. 265). 
said that, in proportion to the comparatively small number attacked, there is probably no infection or contagious disease in this country which produces so much consequent ill-health and disablement as does encephalitis lethargica. My experience has been mostly with children suffering from the sequels of behaviour disorder and post-encephalitic Parkinsonism. Generally speaking, the impression one has received is that the ocular signs and symptoms so commonly present at the onset and during the acute stages of the disease are comparatively rare in the post-encephal. itic stage. ${ }^{2}$ Statistics may be summarized as follows:

Number examined: 174 (boys 113, girls 61).

Average age when first examined: 11 years.

Average date of onset of disease: 3 years previously (6 months to 9 years).

Number of cases with ptosis (one or both sides): 11 6 per cent.)

Number of cases with weak convergence or tendency to divergence: 25 (13 per cent. plus).

Number of cases with concomitant external strabismus: 26 (about 15 per cent.).

Number of cases with concomitant vertical strabismus: 2.

Number of cases with paralysis of internal rectus muscle: 1 .

*Pupils : Verv slight or absent reaction to accommoda tion, 47 (27 per cent.) ; direct light reflex very slight or absent, 23.

Cases having oculogyric crises: 6.

The points to be noted in these statistics are: (1) the infrequency of ptosis; (2) the number of cases with diminished or absent pupil reactions ; (3) the absence of paralytic squint, but the remarkable number with concomitant external strabismus or some form of divergence (28 per cent.) ; and (4) the onset of oculogyric crises.

\section{Ptosis}

Ptosis would appear, therefore, to be the result of lymphocytic inflammation in the acute stage, affecting the third nerve as it passes out from the surface of the crus. This explains why the ptosis disappears as the acute stage dies down. We know, too, that when some only of the third nerve branches are affected by lesions in this situation, the branch to the levator palpebrae is the one most frequently affected. This explanation is supported by the fact that I have never seen an example of Benedikt's syndrome in the numerous chronic encephalitics in whom the red nucleus appears to be affected.

\section{Abnormalities of the Pupil}

The pupil abnormalities are due to active disease of the third nerve nucleus or of its connexions. Some patients developed abnormal pupils two or three years after I had noted them as normal. Arising in the occipital cortex, there are some fibres in the optic radiations which take an opposite course to the majority, and become detached from the optic tract to pass through the crus to the third nerve nucleus. These may be considered as afferent branches to the sphincter pupillae and ciliary muscle. As the substantia nigra and the tegmentum are areas particularly affected in chronic encephalitis, ${ }^{3}$ these fibres may well be affected in this part of their course. We have to remember, also, that the size of the pupil is the resultant of opposing forces actuated by the sympathetic and third nerve, and that in chronic epidemic encephalitis the sympathetic system is in an irritable and

* Note: None of these patients were having drugs producing mydriasis. Only one case showed definite signs of previous congestion of an optic disk, an observation which supports the statement already made as to the rarity of disk changes in the disease. Six patients had oculogyric crises. partially uncontrolled state as the result of lesions in the corpus striatum and optic thalamus. Lastly, convergence is largely a voluntary act and a late development in the history of the human eyes, and the higher control of the lower centres in the mid-brain is markedly affected in this disease.

Herein to some extent may lie the explanation of the form of squint common in these children. Binocular vision is not firmly established until childhood is well advanced, and in the presence of weak convergence these children tend to get concomitant external strabismus.

\section{Oculogyric Crisis}

The oculogyric crises are peculiar to the Parkinsonian form of this disease. In all the cases I have seen there has been spasmodic conjugate movement of the cyes upwards, commonly slightly to one or other side, lasting from a minute to many hours. The patient is conscious of the condition and finds it distressing. He may tell you that his eyes go up and he cannot get them down, or that they won't stay down, as sometimes by an effort he can bring them down for a moment. In a bad Parkinsonian case which I have observed in an adult, the eyes were completely rolled up, the jaw was fixed, with the mouth open, the head remained firmly set in the Parkinsonian forward position, and there was a respiratory crisis, making the patient cyanosed. In some patients the eyes do not go right up, but get fixed only slightly above the horizontal, or complaint is made of staring and that the eves get fixed on an object. It is important for these mild cases to be recognized, and for their symptoms to be remembered by ophthalmologists and not considered hysterical or " functional," especially as these patients tend to be emotional. These crises come on from one to eight years after the original acute illness, and may occur in persons leading more or less normal lives," and so be the first clue to the onset of the post-encephalitic state. ${ }^{5}$ Hence their extreme importance in diagnosis and prognosis, as sooner or later definite Parkinsonism develops. In two adult cases which I examined, the fixing of the eyes was the chief symptom in the early stages. Periodic outbursts of uncontrolled behaviour, either of excitement or grief, may be associated with the crises in adolescents. ${ }^{6}$ Such cases can usually be much controlled by hyoscine or stramonium, whereas this is not so in the acute behaviour disorders not associated with the ocular crises. The onset of the crises points to an activity of the disease, and would appear to indicate danger of psychotic symptoms. In a series of 136 cases which eventually had to be certified, 17 per cent. had oculogyric crises. ${ }^{7}$

It is impossible in a short time to discuss fully the site of the lesions causing these weird attacks. We can see, however, how they fit in with the general conception that many of the appearances of the post-encephalitic state are ląrgely " release" phenomena. Professor Elliot Smith, in his address on the evolution of the instruments of vision at Oxford, ${ }^{14}$ showed a photograph of an anthropoid ape. The semi-erect stance, with the head and shoulders bent forwards, with the arms hanging extended so that the hands were level with the knees, the expressionless face with the dropped lower jaw, together vividly reminded me of the general appearance of many adolescents with severe Parkinsonism." In this disease the mid-brain ${ }^{8}$ (particularly the substantia nigra and the corpus striatum, closely connected by the strio-nigral tract ${ }^{9}$ ) shows most signs of the disease, and so the connecting links for central control over the extra-pyramidal system are affected. Also, irritative lesions of the more primitive centres ${ }^{10}$ in the basal nuclei may occur at the same time as the more recently developed neo-striatum is deficient in controlling them. "Dr. Edwin Bramwell ${ }^{11}$ recently said 
that he knew of no case where a fit characterized by upward or downward movemint of the eyes was associated with disease of the rerebral hemispheres. There appears to be an innate co-ordinated upward movement of the eyes associated with the emotions, and related to a centre at a lower level. The eyes roll upward in sleep, and I have noted how in infancy, at an age when the vegetative centres are apt to take control, the eyes roll up in convulsions caused by gastro-intestinal irritation.

\section{Other Ocular Symptoms}

Other ocular symptoms noted among my patients are excessive blinking, fluttering of the eyelids, and difficulty in moving the eyes in a vertical direction when told to do so. I do not consider these typical of chronic encephalitis, but rather characteristic of the overemotional state of the patients who show them. We have all observed them in emotional or nervous subjects - the act of blinking being closely related to the mental tension of the subject at the time. ${ }^{12}$ Bradykinesis of Parkinsonism is often seen in slow carrying out of the normal voluntary movements of the eyes, and sometimes adds to the difficulties of reading.

\section{Effects of Hyoscine and Stramonium}

I now come to the last part of my subject-namely, the visual disabilities caused by hyoscine or stramonium in the treatment of Parkinsonism. Often ten times the pharmacopoeial dose of stramonium is given, resulting in very marked improvement in the Parkinsonian rigidity. ${ }^{13}$ Many of the patients, however, complain greatly of their inability to read, and of being worried by bright light, due to paresis of accommodation and dilatation of the pupils. Roughly speaking, the stramonium effect as regards accommodation for reading distance is counteracted by $a+1.50$ to 1.75 lens, and eserine and pilocarpine applied to the conjunctival sac seems to counteract the effect to the extent of 11 dioptres. Thus children and young adults with normal refraction, who do not want to read much, can have their visual disabilities removed by myotics. Presbyopes and hypermetropes need glasses, the latter for constant wear. In the case of children, or of adolescents who are myopes or whose mentality is such that they do not wish to read, or who have disorders of behaviour in which the use of glasses is inadvisable, the use of myotics to prevent dazzle is found the best procedure. I have had a group of twenty patients of all these varieties under various myotics ; three have wished to continue with the use of pilocarpine drops rather than have glasses. The problem is a definite one, as it would appear that these Parkinsonian patients have to continue with stramonium for the rest of their lives.

These are but a few of the interesting points which have appealed to me as an ophthalmologist in dealing with these patients.

I should like to thank Dr. Borthwick, the medical superintendent of the Northern Hospital, and Dr. T. R. Hill, for facilities to examine these patients.

\section{References}

${ }^{1}$ Parsons: Ministry of Health ${ }^{\circ}$ Reports, 1922, No. 11

2 Whittington, T. H.: Ministry of Health Reports, 1928, No. 49

3 Pool: Journ. Neurol. and Psychopathol., July, 1930.

${ }^{4}$ Pardee: Amer. Journ. Med. Sci., 1928, clxxv.

${ }^{5}$ Barkas: Lancet, 1926 , ii.

- Borthwick: Journ. State Med., 1928, xxxvi.

7 McCowan and Cook: Brain, 1928, li.

${ }^{8}$ Wilson, Kinnier: Croonian Lecture, Lancet, 1925.

${ }^{9}$ Rawson, S. W.: Anatomy of the Nervous System, 1931.

${ }^{10}$ Hill, T. R.: Annual Report, Metropolitan Asylums Board, $1928-29$

${ }^{11}$ Bramwell, Edwin: Brain, 1928, li.

12 Ponder and Kennedy: Quart. Journ. Exper. Physiol., 1927.

${ }^{13}$ Worster-Drought, and Hill, T. R.: Lancet, June, 1930.

14 Elliot Smith: Oxford Ophthalmological Congress, 1931.

\section{VESICAL SCHISTOSOMIASIS COMPLICATED BY CARCINOMA}

\author{
BY
}

\section{N. HAMILTON FAIRLEY, O.B.E., M.D., D.Sc.,} F.R.C.P.

ASSISTANT PHYSICIAN AND DIRECTOR OF PATHOLOGY, HOSPITAL FOR TROPICAL DISEASES, LONDON ; LECTURER IN CLINICAL MEDICINE AND APPLIED PATHOLOGY, LONDON SCHOOL OF HYGIENE AND TROPICAL IIEDICINE

Vesical schistosomiasis is a disease with an unenviable history, for parasitic schistosomes may survive for long periods in the portal system, depositing ova from time to time in the pelvic viscera with cumulative effects. Accurate information on the longevity of the worms is limited, but Christopherson' ${ }^{1}$ (1924) recorded some important cases where viable ova were passed eighteen to twenty-eight years after patients had contracted their malady. The case recorded below is of special interest, as the duration of the disease was approximately thirty years, during the last twenty-nine of which the patient resided permanently in England.

A man, aged 50, first came to the out-patient department of the Hospital for Tropical Diseases, London, in October, 1930, complaining of intermittent haematuria of approximately thirty years' duration, which had developed during the South African war. Bilharzial infestation had been suspected.

The patient, who went to the South African war in 1900, was invalided to England in 1902 on account of debility and haematuria, which continued for about two years. Since that time the appearance of blood in the urine and vesical discomfort had been essentially intermittent; he only remembered three severe attacks of haematuria throughout the period, and he was often without symptoms for years. Two months before admission a severe relapse occurred, and since then the haematuria has been persistent, blood clots as large as his thumb being passed in the urine. Originally the haematuria was terminal in onset, but latterly the bleeding continued throughout micturition. The urine, on the first examination, contained albumin and many red blood corpuscles, but no ova. The bilharzial skin test, however, was strongly positive, a wheal of $2.5 \mathrm{~cm}$. diameter developing in a few minutes, with the characteristic pseudopodial-like outrunners and surrounding erythema. The complementfixation test was also strongly positive $(+++)$, quantitative titrations showing a fixation of 12 minimal haemolytic doses of complement. Subsequent urinary examination revealed a few dead terminal-spined ova of Schistosoma haematobium. The serological diagnosis of vesical schistosomiasis was thus confirmed.

At this time the patient was not prepared to come into hospital, though the possibilities of superadded renal disease and vesical carcinoma were pointed out. On November 6th, 1930, he returned for admission, complaining of fever, persistent haematuria with the passage of blood clots, strangury, penile irritation, aching pains across the hypogastrium and in the right groin. Loss of weight had recently been noted, and during the previous fortnight he had suffered from profuse sweats. On admission the temperature was $100^{\circ} \mathrm{F}$., the pulse 110, and the respirations 22 per minute. Asthma was also present ; it originated during the South African war. and had since been an annual occurrence.

On examination the patient was found to have distressed breathing and an asthmatic facies: Multiple telangiectasis covered the cheeks. Ten round or oval-shaped nodules, varying in size from 0.5 to $1.5 \mathrm{~cm}$. in diameter, hard in consistency and somewhat bluish in colour, were noted in the subcutaneous tissues over the abdomen and chest. Under local anaesthesia one was removed for microscopical examination.

The tongue was furred, the teeth were carious, and pyorrhoea was marked. The chest moved equally on the two sides, but the air entry was poor. A pleuritic rub was heard over the lower part of the right side of the ches $\bar{t}$ anteriorly; and to a lesser degree behind. The heart showed no abnormality, but the peripheral blood vessels were tortuous and thickened. 